greater number of those who were treated for an adequate time. Of the cases belonging to the third category general improvement was brought about in nearly all and in ten the greater part of the lupus was removed and relapses have been so slight that the patients might almost be regarded as cured. In the remaining cases progress has been very slow and the patients are still under continuous treatment. On the whole, notwithstanding a certain number of cases in which complete success may fairly be claimed, we are compelled to repeat that the light treatment is no more permanent in its effects than older methods. Most of our patients have been obliged by repeated slight relapses to return at intervals for further treatment. In several cases the disease has not been eradicated after nearly five years of almost continuous treatment. It should, however, in fairness be stated that a bald summary of results fails to convey an adequate impression of the beneficial effects of the light treatment. If it not seldom fails to cure it always mitigates the disease and this it does with far less pain and incomparably less distressing after-effects than the severer methods which were formerly the only resource of medicine. It has not indeed made these methods obsolete but it has greatly restricted their application.

In addition to the uncertainty which it shares with every other known method the light treatment has certain special disadvantages. The chief of these are its costliness and the amount of time required for the production of any lasting effect. The former of these objections may be partly removed by the use of the Finsen-Reyn lamp; the latter must, it is to be feared, always be a serious limitation to the usefulness of the treatment. Few patients can afford to give up months, it may be years, of their lives to a treatment which is always irksome and often, during the periods of inflammatory reaction, painful ; even when the cure is to all appearance complete the patient must still remain under supervision for one, two, or even three years.

On the other hand, the treatment has very decided advantages over all others. The most important of these is that there are no deep scarring and no disturbance of the health, however long the treatment may be continued. On balancing the advantages with the disadvantages it must, I think, be admitted that when every allowance has been made for inevitable failures the advantages of the light treatment are so great as to make them well worth securing even at a cost of considerable inconvenience. It is, however, necessary again to emphasise the fact that the light treatment by itself has a very limited sphere of usefulness. As a rule it is effective only in cases in which the disease is slight and superficial, while the impossibility of applying it to the interior of the nose, which there is reason to regard as the starting point of the disease in a large proportion of cases, still further detracts from its value.

Harley-street, W.

\section{MULTIPLE ABSCESSES OF THE KIDNEY DUE TO ACUTE ASCENDING IN- FECTION OF THE NORMAL URINARY TRACT BY BACILLUS COLI COMMUNIS.}

By HAROLD L. BARNARD, M.S. LOND., F.R.C.S. ENG., ASSISTANT SURGEON TO THE LONDON HOSPITAL.

IT would appear that in exceptional circumstances microorganisms may rapidly ascend the normal urinary tract more often of a woman but sometimes of a man and produce as they go urethritis, cystitis, pyelitis, and, finally, multiple hæmorrhagic abscesses of the kidney. This infection seems to be of a transient character, for the urethritis and cystitis clear up spontaneously in two or three days by which time the kidney shows innumerable points of suppuration, and if only one kidney be affected, and this is promptly removed, the patient makes an immediate recovery without further inflammation not only of the lower urinary passages but even of the wound. It would seem well first to narrate the cases on which these statements are founded. The following is the case which first directed my attention to this subject.
CASE 1. Aoute multiple abscesses of the right hidney due to an ascending infection of the urinary tract; nephrectomy immediate recovery without suppuration. - The patient, a man, aged 27 years, was admitted to the London Hospital on Dec. 30th, 1904. On the afternoon of Dec. 24th he had attended some trade festivities connected with the Christmas holiday and had been to an afternoon performance at a theatre. He assured me that he had drunk moderately and only of coffee and lager beer, but as he was returning home at 8 o'clock in the evening he suddenly passed his urine into his trousers. This incontinence was not attended by any pain and he was quite unable to account for it. He assured me that he had not been indiscreet on this or on any previous occasion since his marriage two years before and that at no time had he suffered from any venereal disease. On the following morning at 10 o'clock he was seized with a sudden desire to pass urine and this was followed by severe scalding pains in the urethra. The desire to micturate became more and more frequent and the pain before and after more and more severe until before evening he was suffering from well-marked strangury and was, as he expressed it " bearing down all the time." His urine wa thick and turbid from the start of the attack and he was feverish and thirsty. On the 26th, the second day of the attack, he first had some pain in the loin on the right side and as the day went on this spread round the flank to the front of the abdomen. He did not vomit but suffered from nausea. The frequency of micturition and the turbid character of his urine continued unchanged. He lost all appetite for food and was feverish and thirsty. On the third day the symptoms were much as before but he was worse. On the fourth and fifth days of the attack the pain in his loin became predominant, whilst the scalding in his urethra, and with it the frequency of micturition, steadily diminished until they were scarcely noticeable. The urine, however, remained turbid and his general condition was much worse.

When the patient was admitted to the hospital on Dec. 30th his temperature was $102^{\circ} \mathrm{F}$, and during the next two days it oscillated between $101.5^{\circ}$ and $102^{\circ}$ and twice reached $104^{\circ}$ His pulse varied from 90 to 112 beats per minute and was bounding in character. He was pale and anæmic and looked thoroughly septic. $H$ is urine was acid in reaction, of specific gravity 1018, and contained an eighth of albumin when boiled and allowed to settle. Under the microscope it was found to be full of well-formed polynuclear leucocytes unlike ordinary urinary pus cells. There was no excess of mucus and only one or two red corpuscles were seen. No casts could be found. Unfortunately no bacteriological examination was made. The urethra was examined and there was no purulent discharge although its lips were red and somewhat everted. The cords and testicles were normal and a rectal examination showed nothing amiss with the prostate, vesiculæ, or recto-vesical pouch. On examining the abdomen the upper part on the right side in front, behind, and laterally was exquisitely tender. Deep palpation was difficult but a swelling was made out behind the upper half of the ascending colon. When most ciearly defined this appeared to be the lower end of an enlarged kidney, but at other times it resembled a "recto-colonic" abscess of appendicular origin. There appeared to be no peritonitis. The left kidney was not palpable or tender. The spleen was not enlarged. The heart and the lungs were normal and no source of infection could be found in the form of acne, boils, or a sore throat.

I had previously seen two cases of appendix abscesses which burst into the urinary tract and discharged similar pus in the urine and one of these was retro-colonic in position. I was therefore in doubt whether this was a similar case or an acute infection of the right kidney.

On Jan. 2nd, and on the eighth day of the attack, I cut down on the lump in the loin, placing the centre of my incision over the reflection of the peritoneum so that I could deal with either an appendix abscess or a kidney. The peritoneum in the front part of my incision was normal and I was fortunate enough to encounter the normal appendix in an external position without having to open the abdominal cavity. I then prolonged my incision backwards and upwards and stripped forward the peritoneum and colon. The kidney at once came into the wound and on tearing through its fatty capsule it was found to be about twice its normal size and intensely congested. Its surface was covered with a great number of subcapsular hæmorrhagic spots (Fig. 1); the larger and apparently older of these showed 
yellow suppurating centres. At the time I regarded them as infarcts the result of a shower of minute infective emboli but $I$ argued that the man could not recover with such a kidney in his body and I must risk the other kidney being similarly affected but in a lesser degree, although there was

FIG. 1.

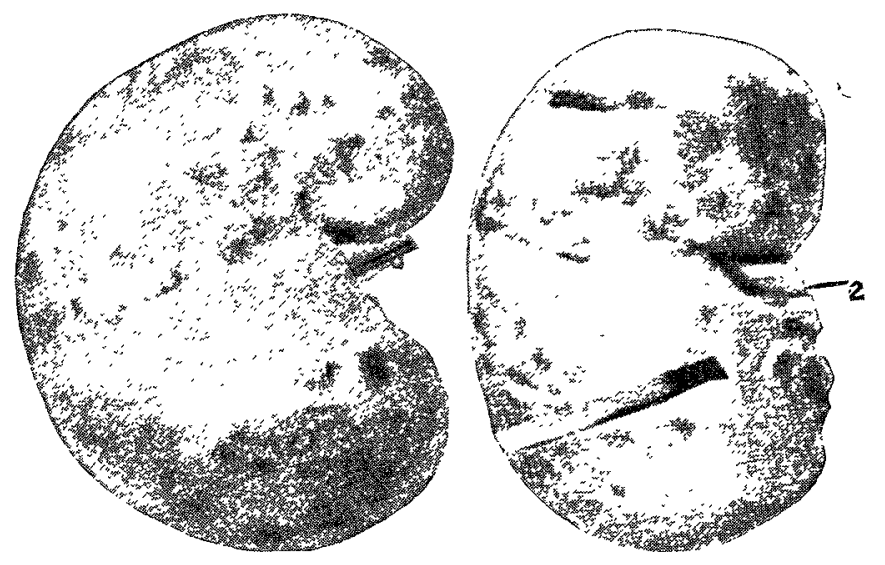

The two halves of the kidney removed in Case 1. The capsule has been stripped off. A great number of raised hæmorrhagic are $\mathrm{s}$ are seen of the size of a small pea. The centres of the larger ones are yellow and purulent.

no evidence of this. I accordingly enucleated the kidney, ligatured the ressels, and removed it, dividing the ureter as low as possible. A drain was inserted. The muscles were united by buried catgut sutures and the skin with continuous catgut.

FIG. 2.

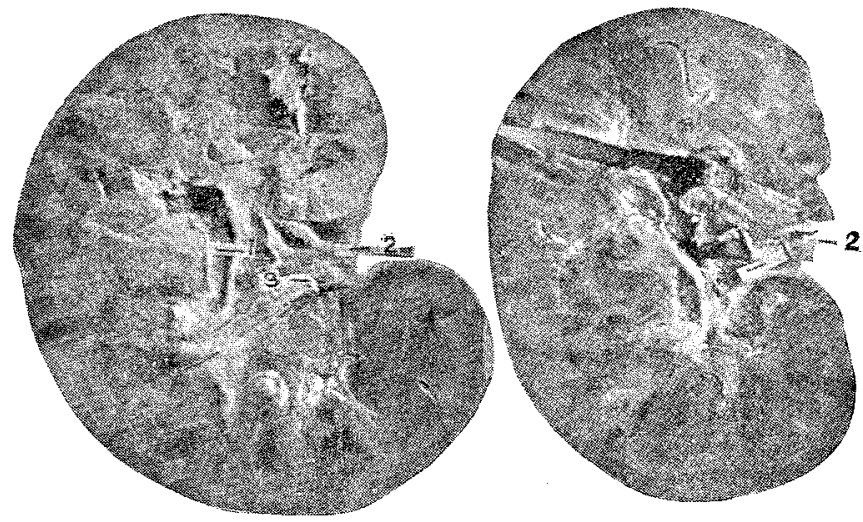

The same kidney in section. The spots on the surface are seen to be the bases of wedged-shaped areas, the auices of which run in towards the pyramins. The en,es of these areas are black and ham

The patient's recovery was immediate and complete. The temperature reached normal at 8 o'clock the same night and only once next day rose above $99^{\circ}$, after which it never again reached this point. The pus disappeared from the urine and leucocytes could only be found by the aid of the centrifuge. The pulse was 72 per minute on the day after the operation and the patient at once lost his septic appearance. The tube was removed from the loin on the fourth day and the wound healed without suppuration.

On dividing the kidney vertically from the cortex to the pelvis the condition shown in Fig. 2 was found. The spots on the surface were seen to be the bases of wedge-shaped hæmorrhagic areas running into the pyramids. The central parts of those wedges were pale, necrotic, and suppurating. Submucous hæmorrhages were found at several points in the pelvis of the kidney. The renal artery and vein were carefully dissected out but no embolus or thrombus could be discovered. Sections were cut by the late Dr. G. R. Slade from a wedged-shaped piece passing through some of the largest patches of suppuration shown in Figs. 1 and 2 . Some were stained with hæmatoxylin; others with methyleme blue and by Gram's method. No micro-organisms could be demonstrated. The areas of inflammation were found to be in the bundles of tubules forming the lobules and not around the vessels between them. No interlobular ressel was thrombosed, only the small twigs which entered the necrotic areas. The inflammation appeared to have started in the tubules, the cells lining which were swollen, stained badly, and in the centre of the areas were entirely disintegrated.

The microscopical sections, the submucons pelvic hæmorrhages, and the history of the onset with urethritis and cystitis seem to leave no doubt that this was an acute ascending infection of the urinary tract. I think it probable that a more careful search would have revealed casts in the urine.

I am indebted to Mr. Hugh M. Rigby, assistant surgeon to the London Hospital, for the following account of his case.

CASE 2. Acute nephritis. - The patient was a woman, aged 35 years, who was admitted to the London Hospital on Oct. 30th, 1903. She had been ill for five days before admission. The first symptom noticed was increased frequency of micturition with severe burning pain during the act. This was followed by pain in the back and the right side and the lower part of the abdomen. The urine passed was of a high colour but did not appear to contain blood She had previously been in good health. As the pains and her general condition became gradually worse she went to the hospital.

On admission the patient, a well-developed woman, looked ill and appeared to be in a good deal of pain. The tongue was furred. Her pulse was 100 per minute, regular, and of full volume. Her temperature was $102 \cdot 5^{\circ} \mathrm{F}$. She com. plained chiefly of pain and tenderness in the right side of the abdomen and right lumbar region. On examination of the abdomen a large tender swelling could be easily felt in the right hypochondriac and lumbar regions; it was in the position of a renal tumour and did not move freely on respiration. There was not marked bulging of the loin but the muscles were rigid. There were no external signs of suppuration. The kidney on the left side could not be felt The urine on examination was found to contain a small amount of blood and pus. It gave an acid reaction and was of specific gravity 1020. A microscopical examination showed the presence of numerous granular and blood casts. Unfortunately a bacteriological examination was not made. On the evening of the day of admission the patient had a rigor, her temperature being $104^{\circ}$. On Oct. 31st, as her condition had not improved, an exploratory operation was made as follows. An incision was made in the right lumbar region and the kidney was exposed. The fascial capsule and kidney fat were found adherent and inflamed. The kidney itself was greatly enlarged. Its surface was deeply congested in places and of a dark purple colour. On the upper half of the convex border many white spots were seen on the surface. An incision over these revealed suppurating foci extending into the medulla of the kidney substance. It was thought advisable to remove the kidney, which was then done. The vessels were ligatured with silk. The ureter was then examined its walls did not appear tbickened or appreciably diseased, so a ligature was tied round it low down. A drainage-tube was inserted and the wound was closed.

The after-progress of the case was most satisfactory. On the following day the temperature fell to normal and remained so. The bladder symptoms and abdominal pains cleared up completely. Three days after the operation the urine was found to be free from blood, pus, and albumin The wound healed by first intention. The tube was removed on the fifth day.

The naked-eye appearance of the kidney showed intensel acute inflammatory changes all over the medulla and cortex. In many places bæmorrhages were seen. The most noticeable feature was the presence of innumerable radiating foci of suppuration extending from the medulla to the cortex some of these formed definite abscesses evidently acute and necrotic in character. The pelvis of the kidney was acutely congested but not dilated. The main vessels of the organ were apparently normal. A microscopical examination revealed a typical acute tubular nephritis; everywhere there was marked round-celled infiltration in the intertubula tissues. The cells lining the tubules were much swollen and granular and in some parts were undergoing acute disin tegration. The section showed no evidence to support the theory that the infection was hæmatogenous. The small vessels were thrombosed but the infiltration of the tissues surrounding them was not more marked than in other part of the field. No micro-organisms were seen.

The following case was rugarded by Dr. G. E. Brewer as one of multiple infective emboli of the kidney. The case is, however, identical with the others recorded and Dr. Brewer was apparently unaware that wedge-shaped areas of 
suppuration in the kidney may arise from an ascending infection of the uriniferous tubules as well as from infarction of the vessels. When an embolus is the cause of the wedge, however, the area is interlobular and the apex terminates in the intermediate zone and between two pyramids. When the wedge is produced by an ascending infection it is intralobular and the apex passes down into a pyramid. Professor Sundberg described this distinction very clearly in 1899 in his description of Professor Lennander's specimen (Case 4) and before reading his description I deseribed an exactly similar condition in my case (see Case 1). It is, moreover, incredible that a myriad of tiny emboli should enter one kidney, should avoid the other and every other organ in the body, and that there should be no origin of sepsis and no septicæmia if the case was really hæmatogenous. I think for these reasons that Dr. Brewer's case may be justly included in this series.

CASE 3. Acute hamatogenous infection of the kidney. ${ }^{1-}$ The patient was a woman, aged 22 years. The case was at first thought to be acute appendicitis. Five days before Dr. Brewer saw the patient she was suddenly seized with intense pain in the right side of her abdomen. This was followed by nausea, vomiting, chills, and fever. There is no note of the presence or absence of frequency of micturition or of scalding. On admission to the Roosevelt Hospital her temperature was $104^{\circ} \mathrm{F}$. and her pulse-rate was 130 per minute. Tenderness was present over the right upper abdomen covering the region of the gall-bladder in front and extending round the side to the costo-vertebral angle behind. It diminished below towards the region of the appendix. Moderate rigidity of the abdominal wall was present over the same area. Per vaginam the right Fallopian tube was found to be a little enlarged and tender. The urine contained albumin, hyaline and granular casts, and a few pus cells. Cystoscopic examination revealed nothing. On catheterising the ureters more pus cells were found in the urine from the right kidney than from the left. The leucocytosis was 18,000 white corpuscles per cubic millimetre. During the next three days the temperature varied from $103^{\circ}$ to $105^{\circ}$. On the fifth day, as the patient was becoming more septic and prostrated, an exploratory laparotomy was decided on. Nothing abnormal was found in the gallbladder, the liver, the cæcum, or the duodenum. The right kidney was thought to be enlarged and was exposed through a lumbar incision. The areolar tissue and fatty capsule were cedematous. The kidney when exposed was deeply congested with numerous subcapsular hæmorrhages. It was incised and the cortex was found to be the seat of numerous "infarcts." A hasty nephrectomy was performed. The patient's temperature dropped in six hours from $105^{\circ}$ to $97.5^{\circ}$ and during the following two days was most of the time subnormal. Her convalescence was rapid. The secretion of urine was from the first satisfactory.

The case was of interest, Dr. Brewer said, for the reason that the lesion was undoubtedly a secondary one due to blood infection and was apparently the only lesion of any importance in the body furnishing all, or nearly all, of the toxins which gave rise to the patient's grave symptoms. In reply to a question Dr. Brewer said that no cultures were made. Dr. Charles H. Peck, who had seen the case with Dr. Brewer, said that the subsidence of the symptoms after the removal of the kidney was very striking.

Dr. Brewer has also recorded two other cases which probably belonged to this class but the notes are too brief to be certain of their identity. ${ }^{2}$

For the following cases I am indebted to Dr. Ernest WVard. my late house surgeon, who has rery kindly searched the entire literature from 1895 to 1905 for them.

CASE 4.-Splitting of the kidnfy in acute pyelonephritis. ${ }^{3}$ The patient, a woman, aged 46 years, was admitted to hospital on Oct. 28th, 1899, and discharged on March 16th, 1900. She had suffered for 14 years with a nerventieber. The onset was sudden, with frequency of micturition accompanied by burning pain, about 14 days before admission and she steadily got worse. On admission there was no sign of kidney infection. The urine was of specific gravity 1012, neutral in reaction, and contained pus cells and some albumin tut no casts. Streptococci and a short bacillus were found.

1 G. E. Brewer: Transactions of the New York Surgical Societ, Oct. 26th, 1904, reported in the Annals of Surgery, December, 1904, p. 1010.

Medical Record, New York, 1905, vol. lxvii., p. 241
On Nov. 2nd the temperature rose to $39 \cdot 4^{\circ} \mathrm{C}$. and the patient felt ill and tenderness appeared over the right kidney. There were right lumbar pain and vomiting on Nov. 4th. The operation was nephrotomy, splitting, and partial resection. The patient made a good recovery and was out of bed on Nov. 13th. A fistula, however, persisted, which was closed by a plastic operation on March 1st. She subsequently passed through an acute pneumonia and nephritis and recovered. The capsule of the kidney was cedematous. Yellow flecks were seen occupying two areas on the kidney cortex. The ureter was thickened and the peri-ureteral tissue was injected. Yellow stripes inside the kidney corresponded to the areas of flecks on the surface. This was the part resected.

The macroscopical description of the parts removed by Professor Sundberg was as follows. The specimen consists of half a pyramid with its cortex bounded by a part of a column of Bertini. The apex of the papilla is pale grey. Higher up are seen two yellowish grey stripes in the direction of the canals surrounded each by a thin reddish-brown hæmorrhagic infiltrated edge. After a course of half a centimetre in the cortex these stripes change into a much altered tissue area, so that they form a wedge-shaped area with the broad base on the outside near the surface. The whole altered tissue reminds one strongly of a hæmorrhagic infarct, but it has its apex in the upper part of a papilla. The cut surface of this area is dark and black, like an infarct, and passing outwards from it are streaks which here and there extend to the surface. Some of these streaks on section are as large as pins' heads and greyish-yellow and exude thin pus, especially one row of small collections immediately beneath the capsule. The surface of the specimen is dry and firm in consistency. The whole of the group forms an area five centimetres wide, surrounded with blood infiltration.

In the pathological institute it was reported that a pure culture of bacillus coli communis was obtained. Microscopical preparations showed no other organism in the kidney. There were pus infiltration and numerous abscesses. In the interstitial tissue were cedema and fresh blood. The renal epithelium was swollen. The canals contained numerous pus cells. The diagnosis was suppurative nephritis.

Lennander records in the same paper five further cases in which multiple abscesses of the kidney were treated by splitiing, partial resection, and drainage. All were operated on within six days of their onset and a similar condition of the kidney was found and in several pure cultures of bacillus coli communis were isolated. I do not, however, propose to narrate them here, as in every case some obstruction of the urinary passages existed or long-standing bladder disease for the most part due to urethro-vaginal fistulæ.

This group of cases is, however, interesting as a connecting link between the well-known surgical kidney due to dilatation and subsequent infection of the urinary passages and the very rare class of case to which I wish to draw attention in which a very virulent form of bacillus coli communis is able to ascend normal urinary passages and to produce multiple miliary abscesses of the kidney. Lennander pointed out that these acute ascending infections of the kidney were often unilateral and that their acute but transient character made it possible to treat them successfully by nephrotomy, partial resections, and drainage, for the disease rapidly tends to clear up. This would, of course, be the only possible course to pursue where both kidneys were affected. On the other hand, Dr. Brewer, who has treated several unilateral cases by Lennander's method, had such unsatisfactory results that he is strongly in favour of nephrectomy.

CASE 5. Splitting of the kidney in acute pyelonephritis with miliary abscesses. ${ }^{*}$-The patient, a woman, aged 23 years, was taken ill suddenly on April 13th, 1901, with strangury and burning pain on micturition. Three days later she had sudden and severe pain in the right loin with pyrexia. Six days after the onset she came under observation when her condition was as follows. Her temperature was $39.8^{\circ} \mathrm{O}$. and her pulse was 130 per minkte. She was not vomiting. The right side of her abdomen was rigid and tender. The right kidney was felt much enlarged. Her urine contained some albumin and a few casts and deposited pus cells but no blood. In spite of the exhibition of urotropin and the "drink cure";

* Dr. Wilms, Leipsic: Mïnchener Medicinische Wochenschrift,


the temperature varied between $39^{\circ}$ and $40.6^{\circ} \mathrm{C}$. and the pyrexia continued. The operation was a nephrotomy. Through a small incision the two halves of the kidney were separated by the finger and a blunt instrument, so avoiding hæmorrhage. The pelvis was opened with scissors. A gauze drain was placed in the pelvis and another around the kidney. All superficial abscesses were opened and drained. The patient did very well. The exudation from the wound was clean. The temperature fell to normal at once. On the third day it rose to $39^{\circ} \mathrm{C}$. on account of a parotitis and reached normal gradually in two weeks. The gauze was gradually extracted during three weeks. The patient was out of bed in five weeks. The fistula closed in eight weeks. The urine was then clear, contained no albumin, and only a few isolated leucocytes. The kidney through the capsule showed a number of small whitish abscesses of the size of peas. In the kidney parenchyma were whitish striped zones which showed the course of the pus infection in the kidney. The pelvis was filled with slightly turbid urine and the vessels were injected. The pelvis was somewhat dilated. The pus in the kidney abscesses gave a pure culture of bacillus coli communis.

CASE 6. Acute renal suppuration. ${ }^{5}$-The patient, a woman, aged 20 years, was admitted to the Roosevelt Hospital on August 6th, 1902. "No history could be obtained in this case that threw any light on the manner of infection and the case is further peculiar in that there were no symptoms referable to the kidney until the day before admission." Just after the noonday meal on the day before admission the patient had frequent desire to urinate, scalding micturition, and small quantities of urine were passed at a time. She also had a sharp shooting pain in the right lumbar and iliac regions, with vomiting and sweating. When admitted to hospital her temperature was $101 \cdot 6^{\circ} \mathrm{F}$. and her pulse was 100 per minute. The abdomen was not distended. The right rectus and quadratus were rigid. Marked tenderness was present over the right kidney and over the appendix. The urine was alkaline, of specific gravity 1030 , and contained a few pus cells. No tubercle bacilli were present. The temperature varied from $99^{\circ}$ to $1026^{\circ}$. On August 8th it shot up to $106^{\circ}$. Leucocytosis was 8000 per cubic millimetre on admission and 9000 per cubic millimetre on August 8th. Nephrectomy was performed on August 9th. Under anæsthesia a mass was felt like an enlarged kidney. Laparotomy was performed through the outer part of the rectus sheath. The left kidney was examined and appeared to be normal. Right lumbar nepbrectomy was then performed. There was little disturbance after the operation. The temperature varied under $101^{\circ}$ for four days and then became normal. The patient was discharged on Sept. 12th with a superficial granulating area. The kidney had no pus around it. It was large and contained numerous cavities filled with pus and there were numerous foci of pus throughout the parenchyma. The ureter was greyish and was filled with pus and appeared gangrenous. Histological examination of the ureter revealed chronic hypertrophic inflammation.

The micro-organism responsible for this disease is apparently the bacillus coli communis. It was found in pure culture in the kidney in Lennander's and Wilm's cases. A bacteriological examination was unfortunately omitted in the other four cases. Moreover, as already stated in a series of five further cases by Lennander in which an exactly similar condition of the kidney was found but complicating pre-existing disease of the lower urinary tract, this bacillus was found in several cases.

It is most interesting to note that the bacillus coli communis displays the same wide variations in virulence in urinary diseases which have always characterised it in the alimentary canal. Of the latter it is a normal inhabitant and is perhaps essential to colonic digestion. When it becomes pathogenic it produces in a crescendo of virulence gastro-enteritis, sloughing and gangrene of the appendix and other structures, and some of the acutest forms of septicæmia. Similarly, in the urinary tract it is found in every degree of virulence. In the condition known as bacilluria it is almost tolerated by the urinary tract with only a slight degree of irritation without any general deterioration of health. In a rather more virulent form it is a common cause of subacute cystitis and pyelitis in such obstructive urinary diseases as urethral stricture, prostatic enlargement, and the pyelitis of pregnancy, but when the obstruction is

5 Joseph A. Blake: Some Problems in the Major Surgery of the Kidneys, with a report of cases, New York Medical News, vol. Ixxxii., removed the inflammation readily subsides. In a more malignant form we find this same micro-organism producing surgical kidneys and destroying life in a few weeks. Finally, as this series of cases has demonstrated, the bacillus coli communis may be so virulent as to ascend a normal urinary tract and proauce hæmorrhagic abscesses in a healthy kidney.

Symptoms.-If it is permissible to sketch the symptomatology of a disease from six cases the symptoms of this would appear to be as follows. A woman, or less frequently a man (in the cases recorded here the proportion was 5 to 1 ), is suddenly seized with a severe scalding pain on micturition and with frequency. These symptoms rapidly increase until a condition of strangury exists. The urine is turbid with leucocytes and desquamated epithelium but as a rule it remains acid or neutral. The condition rapidly becomes worse and pyrexia appears. On the second or third day of the attack the involvement of the pelvis and the kidney is indicated by pain and rigidity in the upper part of the abdomen and the loin, by an increase in the pyrexia, by rigors and vomiting, and by the appearance in the urine of large pear-shaped cells and of casts and of more albumin than can be accounted for by the pus. A few red corpuscles may also be present. During the next two or three days the symptoms of urethritis and cystitis abate and the renal signs become dominant. The patient's condition is one of profound septic poisoning and prostration. The pyrexia is sustained and varies from $102^{\circ}$ to $105^{\circ} \mathrm{F}$. A tumour appears in the region of the infected kidney and the abdominal wall is tender and rigid in the loin, the flanls, and the subcostal region. This tumour was on the right side only in all the cases recorded above and was several times suspected to be an appendix abscess.

I have met no record of a case which has been left without surgical treatment but I suppose death would follow in from 14 to 21 days from septic poisoning. In a few cases a perinephritic abscess might form and be evacuated safely but this must be very rare.

Treatment.-This will depend on whether the infection is unilateral or bilateral. I have not been able to find a record of any bilateral case. When only one kidney is involved it would seem better to expose it through the loin and when it has been seen to be engorged and covered with subcapsular hæmorrhagic abscesses rapidly to excise it. This treatment was followed by immediate cure in four of the six cases recorded above. On the other hand, Cases 4 and 5 recovered with the more conservative measures of splitting, partial resection, and drainage. This method should probably be reserved for slight cases in which the infection is confined to one or two areas and for all bilateral cases. It must be noted, however, that Dr. Brewer lost one or two unilateral cases through following this method and is in favour of nephrectomy in all such cases. After nephrectomy the cure is immediate and complete. The temperature falls to normal in a few hours and does not rise again. The pyuria ceases and the toxæmia disappears. The wound does not suppurate.

General pathology of renal infections.-The paths by which the kidney may be infected are through (1) the blood; (2) the lymphatics; or (3) the urinary passages. Very rarely it may be reached by extension to it of an inflammation, such as from the colon or a retro-colonic appendix abscess.

1. Infeetion of the kidney through the blood stream.--Such infections are seen in some cases of tuberculosis of the kidney and in pyæmia. In tuberculosis the infection may take the form of either an acute miliary infection or of chronic tuberculous foci such as are seen in the strumous kidney of children, but in either case the infection is perivascular and starts as a round-celled infiltration around the vessels of the kidney. In systemic pyæmia, such as in a case of ulcerative endocarditis, the manner of infection is somewhat different although still vascular in origin. Small infective emboli are carried by the blood stream and become impacted usually in the interlobular arteries. Thrombosis and infarction follow and the area, deprived of its blood-supply, is readily invaded by the microorganisms contained in the embolus. Such infections affect both kidneys and as a rule other organs, such as the spleen, the liver, or the brain, and are associated with the general symptoms of pyæmia. It would appear, moreover, that the kidney possesses the function of secreting certain microorganisms, the commonest of which are the bacillus typhosus and the bacillus coli communis. In typhoid 
fever a vast number of bacilli are secreted in this way and render the urine as liable to spread infection as the fæces. In most cases no urinary symptoms are produced, but every degree of nephritis, pyelitis, cystitis, and urethritis may result from the presence of this micro-organism. The condition of bacilluria is due to the presence of bacilli coli communes in the urine where, owing to their motility, they may readily be seen in a fresh specimen of urine under the sixth power of a microscope. The symptoms are seldom severe in uncomplicated cases and consist of frequency and some pain on holding urine and on passing it. It is a not uncommon cause of nocturnal incontinence in children. The urine is acid and nearly clear but usually shows a more or less well-marked cloud on standing a short time. This cloud under the microscope is found to consist of desquamated epithelial cells (hence the name "epithelial cystitis") and the motile bacilli already referred to. Renal signs are seldom present. The condition is nearly always associated with constipation which in some cases is so well marked as to merit the term "coprostasis."

It would appear that in abnormal circumstances and perhaps in connexion with stercoral ulceration the bacillus coli may be absorbed from the alimentary canal and that it is then secreted from the kidney and if in excess it may cause signs of irritation along the whole urinary tract. The proof of this would appear to be that whilst an ordinary infection of the bladder and pelvis of the kidney from below in which the urine remains acid is readily cured by rendering the urine alkaline with potassium citrate these cases of bacilluria are not benefited by this treatment. If, however, the alimentary tract is thoroughly cleared out once a week with calomel they at once yield to the treatment but show a great tendency to relapse as the purgative treatment is discontinued. Malgaigne ${ }^{6}$ was, however, of opinion that bacilluria was due to an infarct of the kidney in spite of the absence in nearly all cases of renal symptoms.

I have discussed this matter at some length because it might be held that in some at present obscure circum. stances a shower of micro-organisms might gain admittance to the blood and without producing constitutional signs might be secreted from the kidney in a similar way, with this difference however-that finding a suitable nidus in the cortex of that organ they might there produce multiple acute abscesses and just such cases as those narrated above. This appears to have been the view taken by Dr. Brewer of Case 3. I do not think, however, that this explanation can be admitted, for it is hard to credit that the microorganism could be secreted from one kidney only and leave the other intact and as very clearly comes out in the majority of the cases recorded above the renal suppuration was preceded for a day or two by acute urethritis and cystitis.

2. Infection of the kidney through the lymph stream.-The anatomy of the lymphatics of the urinary and genital organs is a most fragmentary and uncertain matter. Even a monograph devoted to the subject like that of Poirier, Cuneo, and Delamere, translated and edited by Cecil Leaf, is of very little use in the elucidation of the spread of infectious and malignant growths. Apparently the method of injection is too crude; only short stretches of lymphatic trunks and the glands to which they go can be injected and these are described in fragments as they are discovered. Pathology does not apparently exist for the lymphatic anatomist. He knows nothing of the everyday spread of tubercle from the epidid $y$ mis to the vesiculæ seminales and from these to the ureter and kidney. Apparently there is a subcapsular network of lymphatics which sends branches into the cortex of the kidney and others into the fatty capsule around. This subcapsular network is connected at the hilum with the lymphatics of the ureter and these at their lower end with those of the bladder, prostate, and vas deferens, and through these with the epididymis. It is common knowledge that tubercle may first appear in the epididymis and then infect the vesiculæ and prostate and finally produce cortical deposits in one kidney, even when the mucous membrane of the urinary tract appears normal. It would therefore seem feasible that other micro-organisms might enter these lymphatics in their lower parts and pass by them to the cortex of the kidney, producing there such lesions as have been described in the above cases. Such cases have, indeed, been described as following the operative treatment of urethral stricture. This matter is further complicated by indications that the

6 Archives Générales de Médecine, 1896, vol. ii., p. 722. infection may habitually select certain lymphatic paths. Thus sarcoma of the testicle passes along the lymphatics accompanying the spermatic veins and forms its secondary deposits in the lumbar glands around the hilum of the kidney, whilst on the other hand tubercle starting from the globus major passes apparently along the coverings of the vas deferens to the vesiculæ and prostate and only secondarily involves the kidney. No such point of infection was, however, discovered in any of the above cases.

3. Infection of the kidney along the urinary tract.-I propose to discuss first the means by which the passage of micro-organisms up the urinary passages is normally prevented; then to consider the common ways in which these means are circumvented and in which the ordinary cases of urinary sepsis come about; and, finally, to discuss the instances in which micro-organisms can pass up a normal urinary tract. To the latter class the cases which I have narrated appear to belong.

The means by which the passage of micro-organisms up the urinary traet is normally prevented. - The urinary tract may be regarded as three cavities-the two pelves of the kidney and the bladder-separated from one another and from the surface of the body by long narrow tubes. The urethra undoubtedly guards the bladder from infection. This is proved by the fact that cystitis, apart from gonorrhoea, is so much more common in women who have a short urethra than in men who have a long one. It may, however, be argued that in women the mouth of the urethra is always moist and is frequently exposed to infection from vaginal discharges and I would urge that this is the reason why five out of six of the cases of the disease under discussion were women. The urethra is a narrow tube, so that during the passage of urine there is a good scour. The importance of this for the maintenance of cleanliness in drains and sewers is a subject well understood in hygiene. It is, moreover, provided with a muscular wall which at the end of micturition by peristaltic waves clears its lumen of the last drop of urine and afterwards diminishes that lumen practically to the point of obliteration. Its deep end is closed by the tonically contracted sphincter of the bladder which only relaxes at intervals to once more sweep the tube below it clear of all possible contamination. The proof that the sphincter plays an important part in guarding the bladder from infection is provided by the two common obstructions to the escape of urine from the bladder-viz., stricture of the urethra and enlargement of the prostate. These obstructions differ in that the enlarged prostate obstructs the stream above the sphincter, which is therefore left to guard the bladder as usual. In the case of a stricture, however, the obstruction is below the sphincter, which is, as a consequence, more or less dilated and disordered in its function. Accordingly we find that cystitis is not uncommon in cases of stricture even when no instrument has been passed, whilst in the case of enlarged prostate it is the exception until an instrument has carried the micro-organism past the sphincter. The bladder is undoubtedly more susceptible to infection and bacterial decomposition than the urethra because of the relative stagnation of its contents. Its anatomical arrangement and its muscular coats, however, provide that it shall be completely emptied at frequent intervals. It is probably an acquired adaptation to environment that this frequency is greatly increased when the bladder is infected and that in the condition of strangury the cavity is practically obliterated and the aseptic urine flows from the ureteral orifices in rapidly recurring peristaltic waves over the infected surfaces. The ureters may be regarded as further guards interposed between the bladder and the kidneys to render infection of these vital organs still more difficult. They are even better adapted for this work than the urethra. 'They are longer, narrower, and are swept by a rapid peristaltic wave and completely emptied some 14 times a minute. Their efficiency is proved by the fact that cystitis, though so frequent in women, is seldom followed by surgical kidneys. This is because obstruction is uncommon in them and the ureters are therefore efficient and rareiy dilated. When we do encounter infection of the kidneys in women it is because the function of the ureters has been interfered with by the pregnant uterus, fibroids or carcinoma of the bladder or cervix, or a calculus impacted in the ureter. The pelvis of the kidney is a smaller cavity than the bladder and probably empties itself more frequently. It is, however, very irregular in outline and this accounts for the fact that once 
infected it is longer in freeing itself from that infection than the bladder. Moreover, the urine enters it in a continuous stream from many points and not in intermittent gushes from two as in the case of the bladder or one as in the case of the urethra. The value of an intermittent flow is well illustrated in automatic flushing tanks and cisterns.

The composition of normal urine is unfavourable to the growth of most of the organisms which produce cystitis. Doubtless the acidity of $\mathbf{u}$ ine fulfils other functions, such as keeping earthy phosp'ates in solution, but it is in addition unfavourable to the growth of all the common microorganisms of cystitis except the bacillus coli. If to a prostatic case with alkaline cystitis we give swfficiently large doses of acid sodium phosphate to change the reaction of the urine the micro-organisms will disappear with the exception of the bacillus coli communis and the cystitis will clear up. Dr. A. E. Wright informs me that the bacillus coli grows by preference in alkaline or just neutral media but in common with all this race of bacilli it can grow in acid urine and does not render it alkaline. There are certain indications, moreover, that normal urine contains some factor inimical to the growth of micro-organisms, and this factor belongs perhaps to the class of opsonins which have been found in the serum and other fluids of the body. Dr. Wright believes that such an antibacterial factor exists in the urine and he has kindly written me that he has found on several occasions "that bacteria will grow infinitely more readily in urine of a coli cystitis case than in normal urine. Whereas in the one case 24 hours' incubated urine will contain, say, 300-400 million per c.c., normal urine 24 hours incubated will contain only the same number of thousands." The conclusion to be drawn from this observation is apparently either that the absence of the antibacterial factor leads to the coli cystitis or that the bacillus coli by its growth had exhausted that factor. In any event it would appear that some factor exists which renders normal urine unfavourable for the growth of micro-organisms and assists in maintaining the asepsis of the urinary tract.

The ways in which these means are circumvented and the ordinary cases of urinary sepsis come about.-We have seen that the three great means of protecting the kidneys from bacterial invasion from below are: (1) the small lumen of the urinary passages which secures an efficient scour; (2) the complete emptying of cavities and tubes at frequent intervals of the putrescible urine; and (3) an intermittent flushing from above by a sterile fluid. All these means are circumvented by an obstruction which dilates the urinary passage and cavities. The best marked instance is that of a stricture which has produced retention with overflow in a case in which the ureters and kidneys are already dilated. Here a continuous stagnant chain of urine extends from the kidney to the septic trousers sodden with incontinence. In lesser degrees and earlier cases of stricture the urethra is dilated behind the stricture and this pouch fails to empty itself after micturition. Leakage slowly occurs along the urethra after urine has been passed and the pouch becomes infected. Br this means septic decomposing fluid is held against the mouth of the sphincter vesicr continuously, and as this sphincter has already been dilated and stretched during efforts at micturition it soon allows the passage of these micro-organisms to the urine in the bladder. The bladder, partly through atony of its muscular wall and partly through pouching, never completely empties its cavity and a continuous culture of micro-organisms is thus maintained at the orifices of the ureters. The urine no longer gushes from these in frequent forceful jets. The orifices are dilated. The tubes them. selves are wide, sinuous, and their muscular coat is quite incapatle of emptying their cavities. The sepsis spreads from pond to pond in the varicosities of the ureter and with the greatest ease reaches the irregular and often huge cavity of the pelvis of the kidney. For a time the continuous flow of aseptic urine down the discharging tubules will protect the cortex fron infection, but as the pyramids are de stroyed by pressure and these tubes are consequently shortened, and as interstitial nephritis obstructs their lumen at numerous poirts, the sepsis spreads up them and finally the vital renil substance becomes infected. The history of urinary infection in a case of prostatic enl argement is very similar but, as has already been point sd out, the sphincter and the urethra are here intact and as a rule preserve the urinary tract above the obstruction from infection until the passage of a catheter pushes septic matter bodily past these protecting structures. Then sepsis often spreids with tragic rapidity to both kidneys. Cases of obstruction of the ureters as by calculi are even better protected but not infrequently become infected in women, as in a case that I had under my care in which double pyonephrosis was produced by stones obstructing both ureters and was cured by removing these calculi.

Instances in which nicro-organisms invade a normal urinsry tract. - The commonest example is, of course, the gonococcus but the spread of this micro-organism is often arrested by the sphincter and it seldom passes beyond the bladder. The class of case which we are now discussing appears to me to provide a second example of a micro-organism capable of ascending a normal urinary tract in spite of all safeguards. My reasons for regarding these cases as ascending infections are as follows: 1 . The majority of the cases commenced with urethritis and cystitis. 2. The microscopic examination of the wedge-shaped areas of inflammation in the cortex showed that they were in the lobules and not between the lobules. Moreover, these cases can scarcely be hæmatogenous since: (1) no source of infection was found; (2) the other kidney escaped and all other organs; and (3) their immediate recovery after nephrectomy showed that septicæmia was not present. It is difficult to show that they were not lymphatic in origin for, as has already been pointed out, our knowledge of the lymphatics of the kidneys is obscure. A point of infection in the lower urinary tract would, however, appear essential to a lymphatic infection.

The bacillus coli communis in these cases probably travels along the mucous membrane as the gonococcus does and not along the urine filling the passages as is usually the case in ordinary obstructive infections. The reaction of the body to the invasion appears to be very acute and efficient, for the micro-organism in any part of the lower urinary tract appears to be completely overcome in three or four days. This compares favourably with gonorrhoeal invasion of the urethral mucous membrane or influenzal invasion of the respiratory tract. The spread of the micro-organism is partly by division and multiplication and partly due to its motility, but one cannot help recalling the most interesting experiments of Mr. C. J. Bond of Leicester recorded in his address on Surgery at the meeting of the British Medical Association in July, 1905. He applied sterilised indigo granules to the orifices of various mucous passages and found that the granules were somehow transmitted up these passages in the face of the natural stream of contents and after a day or two could be identified at various distances above. Thus indigo applied to the lips of the uterus was found a day or two later in a pyosalpinx or, in other cases, even in the peritoneum around the ostium abdominale Indigo placed in the rectum was found in a cæcotomy wound three days later, although the bowels had been opened in the interim. Perluaps micro-organisms are in part conveyed from the meatus to the kidney by similar means, whatever these means may be.

Wimpole-street, $\mathrm{W}$.

\section{INTRAVITREOUS HAMORRHAGES.}

By LESLIE PATON, M.B. CaNtab., F.R.C.S. ENG., ASSISTAN'T OPHTHALMIC SURGEON, ST. MARY'S HOSPITAL; AND

W. ERASMUS PARAMORE, M.B., B.C. Cantab.

THE occurrence of large hæmorrhages in the retina and into the vitreous in a seemingly spontaneous fashion is frequently met with in ophthalmic practice. That such hæmorrhages occurred in young people who to all outward appearance were in perfect health was a fact to which $\mathrm{Mr} . \mathrm{H}$. Eales of Birmingham first called attention in the year 1880. Mr. Eales described the cases as occurring in healthy young men. We have given here two cases in young women as they presented clinically the same features and showed the same changes in their blood. We have recently had the opportunity of seeing four such cases and of examining the blood by methods devised by Dr. A. E. Wright, whom we must thank for his kind advice and coöperation in the work. One of us (L. P.) is responsible for the clinical notes while the examinations of the blood were made by the other of us. It will be convenient to begin by giving a short history of the cases from the point of view of the ophthalmologist.

CASE 1.-On Tuesday, Sept. 6th, 1904, a patient (a medical man) came to see me (L. P.) who on the previous Friday 